\title{
Study of the eHWC J1825-134 Region at Highest Energies with HWAC
}

\section{Dezhi Huang, ${ }^{a, *}$ Francisco Salesa Greus, ${ }^{b, c}$ Sabrina Casanova ${ }^{b}$ and Petra Huentemeyer $^{a}$ on behalf of the HAWC Collaboration \\ (a complete list of authors can be found at the end of the proceedings)}

${ }^{a}$ Michigan Techonology University, Department of Physics

1400 Townsend Drive, Houghton, USA

${ }^{b}$ Institute of Nuclear Physics Polish Academy of Sciences,

PL-31342 IFJ-PAN, Krakow, Poland

${ }^{c}$ Instituto de Física Corpuscular,

CSIC, Universitat de València, E-46980, Paterna, Valencia, Spain

E-mail: dezhih@mtu.edu, sagreus@ific.uv.es, sabrina.casanova@ifj.edu.pl, petra@mtu.edu

eHWC J1825-134 is one of the brightest Galactic $\gamma$-ray sources above $50 \mathrm{TeV}$ observed by High Altitude Water Cherenkov $\gamma$-Ray Observatory (HAWC). Detailed morphological studies have revealed a new point-like source inside this region with a spectral energy distribution extending beyond $200 \mathrm{TeV}$ without any cutoff. These very-high-energy $\gamma$ rays emission can originate from leptonic or hadronic processes. The new point-like source is located in a region containing PWNe and a high density giant molecular cloud [MML2017]99. If the source emission is associated with the hadronic scenario the $\mathrm{TeV} \gamma$ rays may have been produced by cosmic rays colliding with ambient gas. If this were the case, eHWC J1825-134 is an indicator of the existence of a galactic PeVatron in the region that accelerates particles up to PeV energies.

$37^{\text {th }}$ International Cosmic Ray Conference (ICRC 2021)

July 12th - 23rd, 2021

Online - Berlin, Germany

\footnotetext{
*Presenter
} 


\section{Introduction}

Ever since the Austrian-American scientist, Victor Hess detected cosmic rays over a hundred years ago, the origin of these charged particles has been a puzzle for mankind. People believe cosmic rays up to the "knee" are of the galactic origin. This means inside our galaxy extremely powerful accelerators are accelerating charged particles beyond PeV, the so called PeVatrons.

Sitting on the Sierra Negra volcano near Puebla, Mexico at the altitude of 4100 meters above the sea level, the HAWC Observatory observes $\gamma$ rays produced by high energy particles accelerated by these powerful sources in the Northern Hemisphere. The high duty cycle, large field of view, and excellent sensitivity above $10 \mathrm{TeV}$ make HAWC one of the greatest instruments to study the galactic $\gamma$-ray sources[1]. Additionally, newly installed outrigger array increases HAWC's sensitivity by a factor of 4 at highest energies. This upgrade will be crucial for studying PeVatrons at the highest energy range.

Using 1038 days of HAWC data, there are 9 eHWC sources with the emission beyond $56 \mathrm{TeV}$ [2]. Among those high energy galactic $\gamma$-ray sources, the most bright source eHWC J1825-134 stands out with the emission above $100 \mathrm{TeV}$.
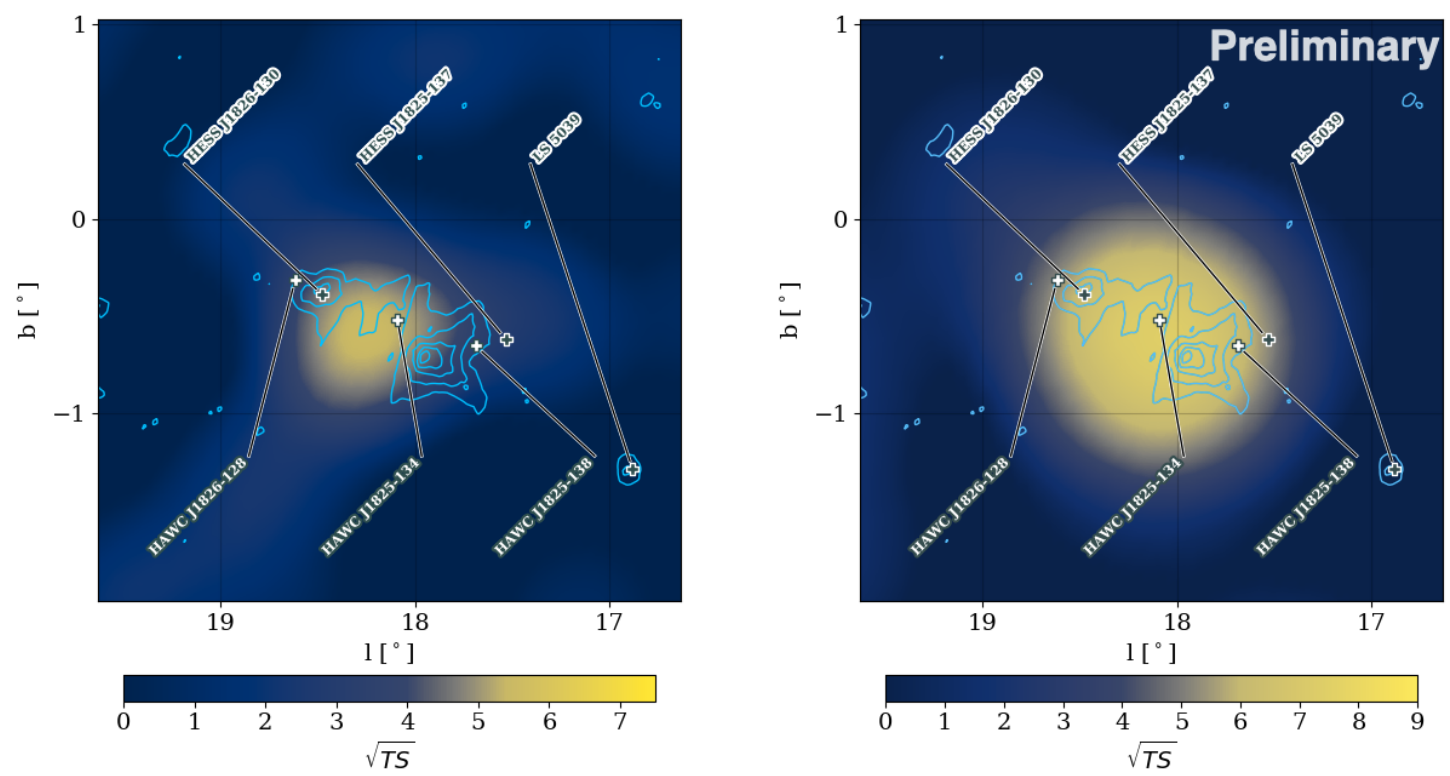

Figure 1: Significance map of eHWC J1825 region with energy greater than $177 \mathrm{TeV}$. The blue contours corresponding to H.E.S.S. excess at 20, 35, 50, 60, and 70 counts with energies beyond $10 \mathrm{TeV}$ [3]. Significance maps are made assuming a point-source hypothesis and a powerlaw spectrum with -2.6 index.

\section{Analysis of eHWC J1825-134 Region}

To do a detail study of eHWCJ1825-134 region, the maximum likelihood analysis was performed using the Multi-Mission Maximum Likelihood (3ML) [4] and Hal plugin[5] [6]. The result was reported in a recent HAWC publication [7]. 
From this study we found the eHWC J1825-134 region can be well described by two extended sources HAWC J1825-138 and HAWC J1826-128 plus one point source HAWC J1825-134 on top of the galactic diffuse emission (GDE). The two extended sources are likely to have known $\gamma$-ray counterparts which are HESS J1825-137 and HESS J1826-130 [8] , respectively.

The spectral energy distribution (SED) analysis shows both extended sources are well fitted with the power-law with an exponential cutoff. Both SEDs start cutting off at below $30 \mathrm{TeV}$. However, the new point source shows no indication of any cutoff up to $200 \mathrm{TeV}$ with a best fit power-law index at $2.28{ }_{-0.12}^{+0.12}{ }_{-0.04}^{+0.1}$, the uncertainties are statistical and systematic, in that order. Located close to our new point source HAWC J1825-134 is a young star cluster, [BDS2003]8, with

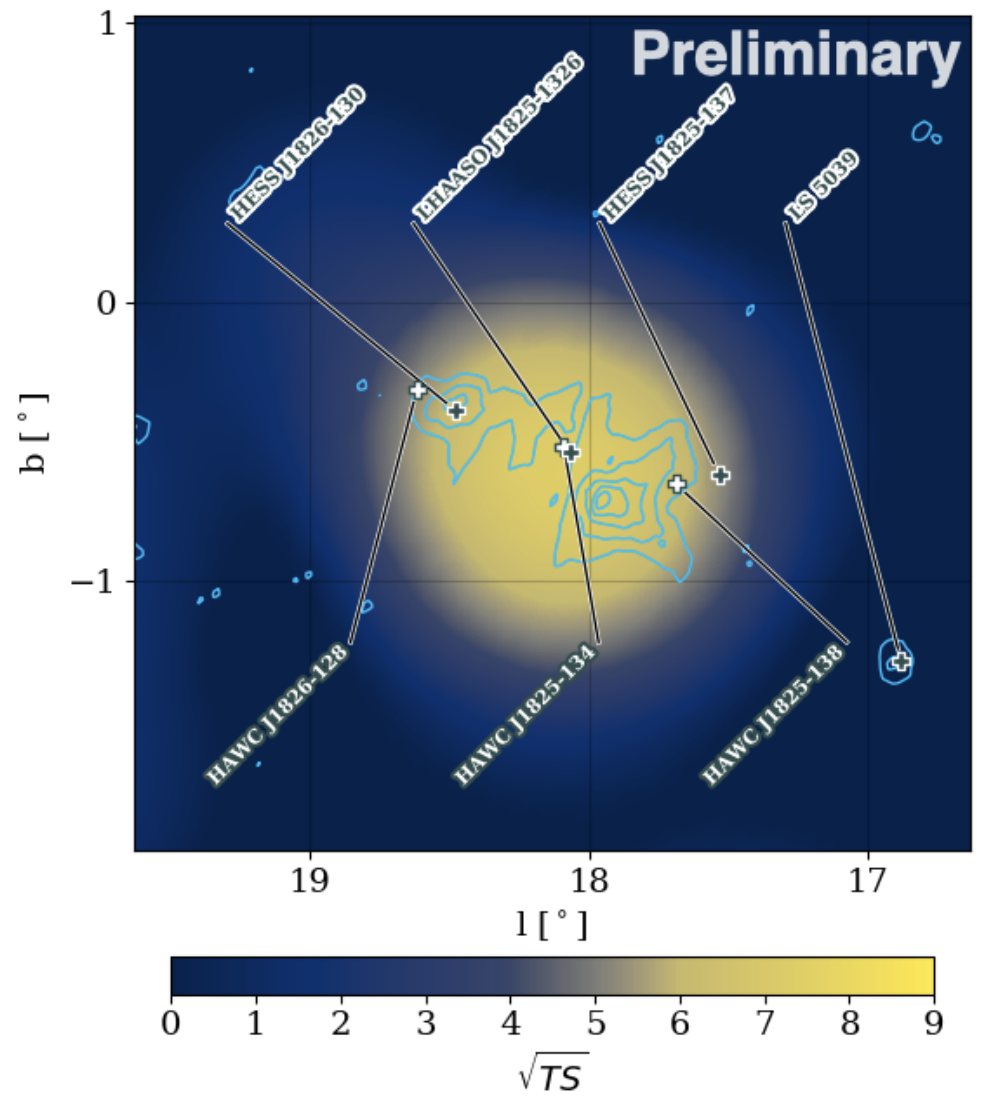

Figure 2: Preliminary Pass5 Significance map of eHWC J1825-134 region with energy greater than $177 \mathrm{TeV}$ include the location from LHAASO publication [12].The blue contours corresponding to H.E.S.S. excess at $20,35,50,60$, and 70 counts with energies beyond $10 \mathrm{TeV}$ [3]

the age of $\sim 1$ million years and a distance of $4 \mathrm{kpc}$ from the Earth [9] [10]. The total energy budget can reach $1.5 \times 10^{52} \mathrm{erg}$ [11]. We only need a small fraction of this energy to accelerate a proton up to $\mathrm{PeV}$. The $\mathrm{PeV}$ protons confined in the region likely collide with the giant molecular cloud [MML2017]99 to produce the emission observable by HAWC. From our analysis of eHWC J1825-134 region supports that young star clusters are galactic PeVatron candidates to contribute in the cosmic-ray flux up to the knee. 


\section{High Energy Point Source HAWC J1825-134}

The preliminary HAWC Pass5 analysis shows that the new point source HAWC J1825-134 becomes more significantly in our map. With this new Pass5 data set we observe $7.3 \sigma$ at the energies beyond $177 \mathrm{TeV}$. Also, the maximum significance pixel has moved towards to the HAWC J1825-134. We do have a slight worse point spread function (PSF) at this region $\left(0.4^{\circ}\right.$ in the publication and $0.43^{\circ}$ in the new data-set). This will be investigated in a future analysis.

The newly published result from Large High Altitude Air Shower Observatory (LHAASO) [12] confirms our observation around the eHWC J1825-134 region. LHAASO observed the emission at greater than $100 \mathrm{TeV}$ with $16.4 \sigma$ using half of Kilometer Square Array (KM2A) with 308 days of data. They reported the maximum energy from this region reaching up to $420 \mathrm{TeV}$ [12]. Figure 2 shows the significance map made with preliminary Pass 5 data. We can see the location of the point source HAWC J1825-134 almost overlapping with LHASSO J1825-1326. From the HAWC analysis both extended sources start cutting off before $30 \mathrm{TeV}$. Considering LHAASOKM2A has great sensitivity at beyond $10 \mathrm{TeV}$, LHAASO-KM2A's result likely confirms the high energy emission between two extended sources.

In addition to the location, we also compared with the flux from LHAASO. From Figure 3 the LHAASO flux point at $200 \mathrm{TeV}$ agrees with the HAWC J1825-134 spectrum well. Beyond 300 $\mathrm{TeV}$ we do see that the spectrum becomes softer even if we consider the $\gamma-\gamma$ absorption. Since the absorption becomes significant once the energy passes $200 \mathrm{TeV}$, detailed analyses of eHWC J1825-134 from LHASSO and HAWC outriggers will be crucial to study the spectrum beyond 200 $\mathrm{TeV}$.

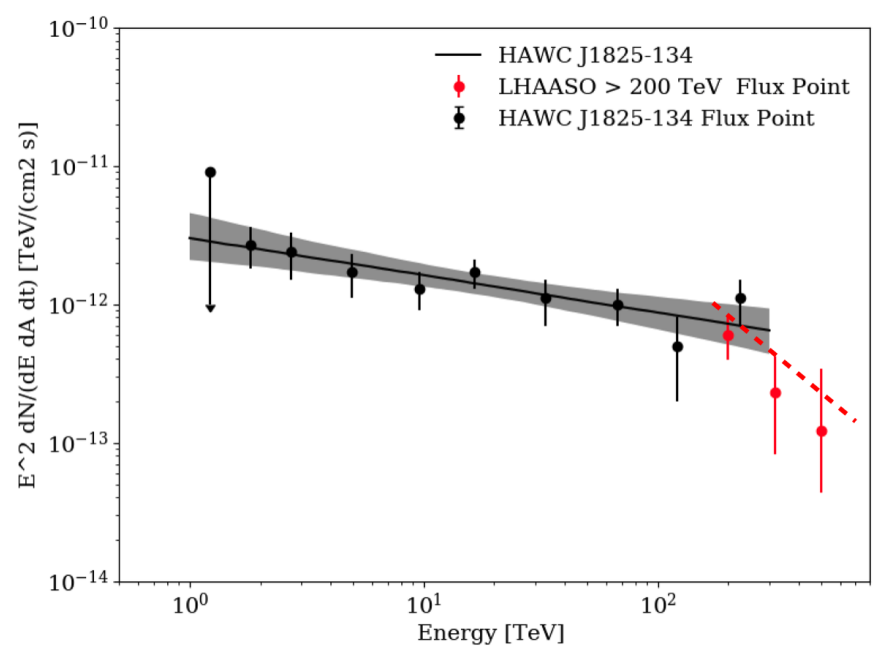

Figure 3: The black spectrum and points are showing the best fit spectrum and flux points of HAWC J1825134. The red data points are LHAASO flux points greater than $200 \mathrm{TeV}$, the dish red line is the estimated LHAASO spectrum after corrected from $\gamma-\gamma$ absorption. 


\section{Energy Dependent Morphology of HAWC J1825-138}

To understand the eHWC J1825-134 region better, we preform morphology study to one of the extended sources HAWC J1825-138 inside this region. A previous result from H.E.S.S. [13] [3] shows there is a clear trend of $\gamma$-ray index becoming softer when the emission moves further away from the PSR J1826-1334. This can be explained through the $\gamma$ rays been produced by electrons up-scattering the ambient low energy photons. The high energy electrons will be cooling faster that will cause the high energy $\gamma$-ray emission to be closer to the accelerator, PSR J1825-1334 in this case.

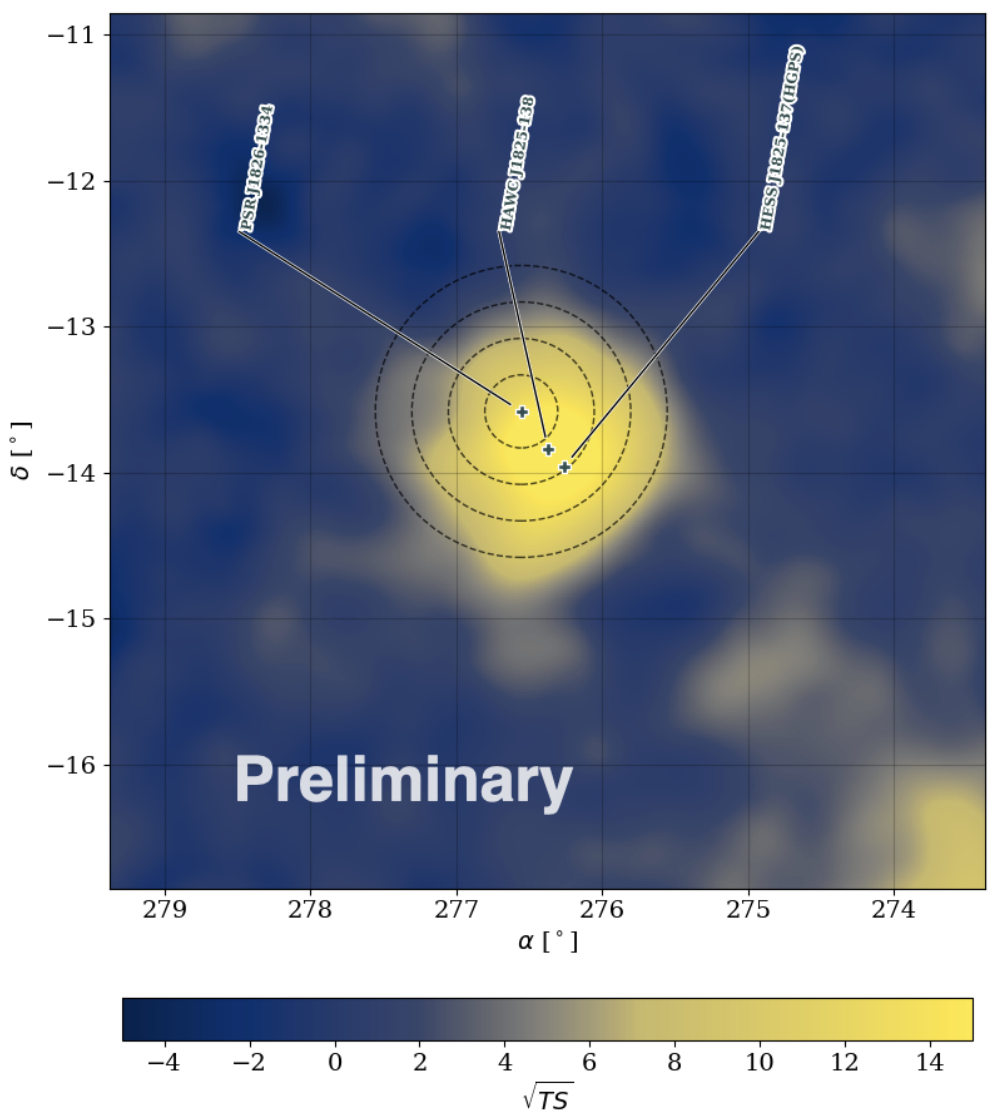

Figure 4: Significance map of HAWC J1825-138 after subtracting HAWC J1826-128, HAWC J1825-134 and galactic diffuse emission.

To do the analysis, first we subtract the best fitted model of the extended source HAWC J1826-128, point source HAWC J1825-134 and galactic diffuse emission from the HAWC data. Figure 4 shows the residual map of HAWC J1825-134 after subtracting the fitted HAWC J1826128, HAWC J1825-134 and GDE. The symmetric Gaussian morphology with $0.47^{\circ}$ width fits the HAWC data well. To cover the most of the emission region we choose four rings centered at PSR 
$\mathrm{J} 1825-1334$, the center ring has $0.25^{\circ}$ in radius, the remaining three rings have extra $0.25^{\circ}$ compared to the inner-most ring.

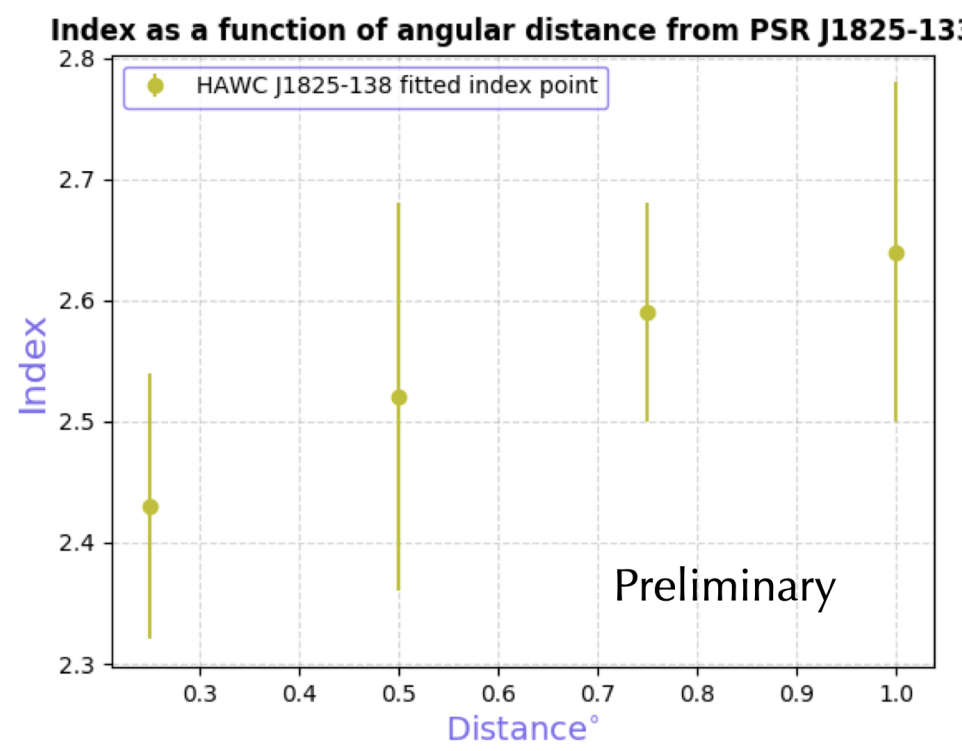

Figure 5: Index as a function of distance from PSR J1826-1334

Then we fit the power-law spectrum inside each ring. The result is shown in Figure 5. We can see the index becomes softer as the emission move further from the pulsar. Although, we do have large error bars with this analysis. More careful analysis is needed using the upcoming HAWC data to identify this trend.

\section{Conclusion}

The studies of eHWC J1825-134 region from HAWC [7] and LHAASO [12] found the $\gamma$-ray emission extended beyond $200 \mathrm{TeV}$. LHASSO's flux point at $200 \mathrm{TeV}$ agrees with HAWC J1825134 best fit spectrum. We do see the last two flux points step down possible caused by $\gamma-\gamma$ absorption. The future analysis that including the HAWC outrigger array will be important at the highest energies.

Also, the energy-dependent morphology of large extended source HAWC J1825-138 shows the potential indicating that the power-law spectrum index becomes softer as the emission move further from the pulsar. A recent publication suggests this extended source could be a hybrid of TeV halo and pulsar wind nebula [14]. The future HAWC analysis will help to solve the puzzles in the eHWC J8125-134 region.

\section{Acknowledgments}

We acknowledge the support from: the US National Science Foundation (NSF); the US Department of Energy Office of High-Energy Physics; the Laboratory Directed Research and Development (LDRD) program of Los Alamos National Laboratory; Consejo Nacional de Ciencia y Tecnología 
(CONACyT), México, grants 271051, 232656, 260378, 179588, 254964, 258865, 243290, 132197, A1-S-46288, A1-S-22784, cátedras 873, 1563, 341, 323, Red HAWC, México; DGAPA-UNAM grants IG101320, IN111716-3, IN111419, IA102019, IN110621, IN110521; VIEP-BUAP; PIFI 2012, 2013, PROFOCIE 2014, 2015; the University of Wisconsin Alumni Research Foundation; the Institute of Geophysics, Planetary Physics, and Signatures at Los Alamos National Laboratory; Polish Science Centre grant, DEC-2017/27/B/ST9/02272; Coordinación de la Investigación Científica de la Universidad Michoacana; Royal Society - Newton Advanced Fellowship 180385; Generalitat Valenciana, grant CIDEGENT/2018/034; Chulalongkorn University's CUniverse (CUAASC) grant; Coordinación General Académica e Innovación (CGAI-UdeG), PRODEP-SEP UDG-CA499; Institute of Cosmic Ray Research (ICRR), University of Tokyo, H.F. acknowledges support by NASA under award number 80GSFC21M0002. We also acknowledge the significant contributions over many years of Stefan Westerhoff, Gaurang Yodh and Arnulfo Zepeda Dominguez, all deceased members of the HAWC collaboration. Thanks to Scott Delay, Luciano Díaz and Eduardo Murrieta for technical support.

\section{References}

[1] Abeysekara, A. U., et al. "Observation of the crab nebula with the HAWC gamma-ray observatory." The Astrophysical Journal 843.1 (2017): 39.

[2] Abeysekara, A. U., et al. "Multiple Galactic Sources with Emission Above $56 \mathrm{TeV}$ Detected by HAWC." Physical review letters 124.2 (2020): 021102.

[3] Abdalla, H., et al. "Particle transport within the pulsar wind nebula HESS J1825-137." Astronomy \& Astrophysics 621 (2019): A116.

[4] Vianello, Giacomo, et al. "The Multi-Mission Maximum Likelihood framework (3ML)." arXiv preprint arXiv:1507.08343 (2015).

[5] Chad Brisbois, Characterizing $\gamma$-ray sources with HAL (HAWC Accelerated likelihood) and 3ML, ICRC2021

[6] Younk, Patrick W., et al. "A high-level analysis framework for HAWC." arXiv preprint arXiv:1508.07479 (2015).

[7] Albert, A., et al. "Evidence of $200 \mathrm{TeV}$ photons from HAWC J1825-134." The Astrophysical Journal Letters 907.2 (2021): L30.

[8] Abdalla, H., et al. "The HESS Galactic plane survey." Astronomy \& Astrophysics 612 (2018): A1.

[9] Brand, J., and L. Blitz. "The velocity field of the outer Galaxy." Astronomy and Astrophysics 275 (1993): 67.

[10] Bica, E., et al. "New infrared star clusters in the Northern and Equatorial Milky Way with 2MASS." Astronomy \& Astrophysics 404.1 (2003): 223-232. 
[11] Crowther, Paul A., and Luc Dessart. "Quantitative spectroscopy of Wolf-Rayet stars in HD 97950 and R136a - the cores of giant H ii regions." Monthly Notices of the Royal Astronomical Society 296.3 (1998): 622-642.

[12] Cao, Zhen, et al. "Ultrahigh-energy photons up to 1.4 petaelectronvolts from $12 \gamma$-ray Galactic sources." Nature (2021): 1-4.

[13] Aharonian, Felix, et al. "Energy dependent $\gamma$-ray morphology in the Pulsar wind nebula HESS J1825-137." Astronomy \& Astrophysics 460.2 (2006): 365-374.

[14] Giacinti, G., et al. "Halo fraction in TeV-bright pulsar wind nebulae." Astronomy \& Astrophysics 636 (2020): A113. 


\section{Full Authors List: HAWC Collaboration}

A.U. Abeysekara ${ }^{48}$, A. Albert ${ }^{21}$, R. Alfaro ${ }^{14}$, C. Alvarez ${ }^{41}$, J.D. Álvarez ${ }^{40}$, J.R. Angeles Camacho ${ }^{14}$, J.C. Arteaga-Velázquez ${ }^{40}$, K. P. Arunbabu $^{17}$, D. Avila Rojas ${ }^{14}$, H.A. Ayala Solares ${ }^{28}$, R. Babu ${ }^{25}$, V. Baghmanyan ${ }^{15}$, A.S. Barber ${ }^{48}$, J. Becerra Gonzalez ${ }^{11}$, E. BelmontMoreno $^{14}$, S.Y. BenZvi ${ }^{29}$, D. Berley ${ }^{39}$, C. Brisbois ${ }^{39}$, K.S. Caballero-Mora ${ }^{41}$, T. Capistrán ${ }^{12}$, A. Carramiñana ${ }^{18}$, S. Casanova ${ }^{15}$, O. Chaparro-Amaro ${ }^{3}$, U. Cotti ${ }^{40}$, J. Cotzomi ${ }^{8}$, S. Coutiño de León ${ }^{18}$, E. De la Fuente ${ }^{46}$, C. de León ${ }^{40}$, L. Diaz-Cruz ${ }^{8}$, R. Diaz Hernandez ${ }^{18}$, J.C. Díaz-Vélez ${ }^{46}$, B.L. Dingus ${ }^{21}$, M. Durocher ${ }^{21}$, M.A. DuVernois ${ }^{45}$, R.W. Ellsworth ${ }^{39}$, K. Engel ${ }^{39}$, C. Espinoza ${ }^{14}$, K.L. Fan ${ }^{39}$, K. Fang $^{45}$, M. Fernández Alonso ${ }^{28}$, B. Fick ${ }^{25}$, H. Fleischhack ${ }^{51,11,52}$, J.L. Flores ${ }^{46}$, N.I. Fraija ${ }^{12}$, D. Garcia ${ }^{4}$, J.A. García-González ${ }^{20}$, J. L. García-Luna ${ }^{46}$, G. García-Torales ${ }^{46}$, F. Garfias ${ }^{12}$, G. Giacinti ${ }^{22}$, H. Goksu ${ }^{22}$, M.M. González ${ }^{12}$, J.A. Goodman ${ }^{39}$, J.P. Harding ${ }^{21}$, S. Hernandez ${ }^{14}$, I. Herzog ${ }^{25}$, J. Hinton ${ }^{22}$, B. Hona ${ }^{48}$, D. Huang ${ }^{25}$, F. Hueyotl-Zahuantitla ${ }^{41}$, C.M. Hui ${ }^{23}$, B. Humensky ${ }^{39}$, P. Hüntemeyer ${ }^{25}$, A. Iriarte ${ }^{12}$, A. Jardin-Blicq ${ }^{22,49,50}$, H. Jhee ${ }^{43}$, V. Joshi ${ }^{7}$, D. Kieda ${ }^{48}$, G J. Kunde ${ }^{21}$, S. Kunwar ${ }^{22}$, A. Lara ${ }^{17}$, J. Lee ${ }^{43}$, W.H. Lee ${ }^{12}$, D. Lennarz ${ }^{9}$, H. León Vargas ${ }^{14}$, J. Linnemann ${ }^{24}$, A.L. Longinotti ${ }^{12}$, R. López-Coto ${ }^{19}$, G. Luis-Raya ${ }^{44}$, J. Lundeen ${ }^{24}$, K. Malone ${ }^{21}$, V. Marandon $^{22}$, O. Martinez ${ }^{8}$, I. Martinez-Castellanos ${ }^{39}$, H. Martínez-Huerta ${ }^{38}$, J. Martínez-Castro ${ }^{3}$, J.A.J. Matthews ${ }^{42}$, J. McEnery ${ }^{11}$, P. Miranda-Romagnoli $^{34}$, J.A. Morales-Soto ${ }^{40}$, E. Moreno ${ }^{8}$, M. Mostafá ${ }^{28}$, A. Nayerhoda ${ }^{15}$, L. Nellen ${ }^{13}$, M. Newbold ${ }^{48}$, M.U. Nisa ${ }^{24}$, R. Noriega-Papaqui ${ }^{34}$, L. Olivera-Nieto ${ }^{22}$, N. Omodei ${ }^{32}$, A. Peisker ${ }^{24}$, Y. Pérez Araujo ${ }^{12}$, E.G. Pérez-Pérez ${ }^{44}$, C.D. Rho ${ }^{43}$, C. Rivière ${ }^{39}$, D. Rosa-Gonzalez $^{18}$, E. Ruiz-Velasco ${ }^{22}$, J. Ryan ${ }^{26}$, H. Salazar ${ }^{8}$, F. Salesa Greus ${ }^{15,53}$, A. Sandoval ${ }^{14}$, M. Schneider ${ }^{39}$, H. Schoorlemmer ${ }^{22}$, J. Serna-Franco ${ }^{14}$, G. Sinnis ${ }^{21}$, A.J. Smith ${ }^{39}$, R.W. Springer ${ }^{48}$, P. Surajbali ${ }^{22}$, I. Taboada ${ }^{9}$, M. Tanner ${ }^{28}$, K. Tollefson ${ }^{24}$, I. Torres ${ }^{18}$, R. Torres-Escobedo $^{30}$, R. Turner ${ }^{25}$, F. Ureña-Mena ${ }^{18}$, L. Villaseñor ${ }^{8}$, X. Wang ${ }^{25}$, I.J. Watson ${ }^{43}$, T. Weisgarber ${ }^{45}$, F. Werner ${ }^{22}$, E. Willox ${ }^{39}$, J. Wood ${ }^{23}$, G.B. Yodh ${ }^{35}$, A. Zepeda ${ }^{4}$, H. Zhou ${ }^{30}$

${ }^{1}$ Barnard College, New York, NY, USA, ${ }^{2}$ Department of Chemistry and Physics, California University of Pennsylvania, California, PA, USA, ${ }^{3}$ Centro de Investigación en Computación, Instituto Politécnico Nacional, Ciudad de México, México, ${ }^{4}$ Physics Department, Centro de Investigación y de Estudios Avanzados del IPN, Ciudad de México, México, ${ }^{5}$ Colorado State University, Physics Dept., Fort Collins, CO, USA, ${ }^{6}$ DCI-UDG, Leon, Gto, México, ${ }^{7}$ Erlangen Centre for Astroparticle Physics, Friedrich Alexander Universität, Erlangen, BY, Germany, ${ }^{8}$ Facultad de Ciencias Físico Matemáticas, Benemérita Universidad Autónoma de Puebla, Puebla, México, ${ }^{9}$ School of Physics and Center for Relativistic Astrophysics, Georgia Institute of Technology, Atlanta, GA, USA, ${ }^{10}$ School of Physics Astronomy and Computational Sciences, George Mason University, Fairfax, VA, USA, ${ }^{11}$ NASA Goddard Space Flight Center, Greenbelt, MD, USA, ${ }^{12}$ Instituto de Astronomía, Universidad Nacional Autónoma de México, Ciudad de México, México, ${ }^{13}$ Instituto de Ciencias Nucleares, Universidad Nacional Autónoma de México, Ciudad de México, México, ${ }^{14}$ Instituto de Física, Universidad Nacional Autónoma de México, Ciudad de México, México, ${ }^{15}$ Institute of Nuclear Physics, Polish Academy of Sciences, Krakow, Poland, ${ }^{16}$ Instituto de Física de São Carlos, Universidade de São Paulo, São Carlos, SP, Brasil, ${ }^{17}$ Instituto de Geofísica, Universidad Nacional Autónoma de México, Ciudad de México, México, ${ }^{18}$ Instituto Nacional de Astrofísica, Óptica y Electrónica, Tonantzintla, Puebla, México, ${ }^{19}$ INFN Padova, Padova, Italy, ${ }^{20}$ Tecnologico de Monterrey, Escuela de Ingeniería y Ciencias, Ave. Eugenio Garza Sada 2501, Monterrey, N.L., 64849, México, ${ }^{21}$ Physics Division, Los Alamos National Laboratory, Los Alamos, NM, USA, ${ }^{22}$ Max-Planck Institute for Nuclear Physics, Heidelberg, Germany, ${ }^{23}$ NASA Marshall Space Flight Center, Astrophysics Office, Huntsville, AL, USA, ${ }^{24}$ Department of Physics and Astronomy, Michigan State University, East Lansing, MI, USA, ${ }^{25}$ Department of Physics, Michigan Technological University, Houghton, MI, USA, ${ }^{26}$ Space Science Center, University of New Hampshire, Durham, NH, USA, ${ }^{27}$ The Ohio State University at Lima, Lima, OH, USA, ${ }^{28}$ Department of Physics, Pennsylvania State University, University Park, PA, USA, ${ }^{29}$ Department of Physics and Astronomy, University of Rochester, Rochester, NY, USA, ${ }^{30}$ Tsung-Dao Lee Institute and School of Physics and Astronomy, Shanghai Jiao Tong University, Shanghai, China, ${ }^{31}$ Sungkyunkwan University, Gyeonggi, Rep. of Korea, ${ }^{32}$ Stanford University, Stanford, CA, USA, ${ }^{33}$ Department of Physics and Astronomy, University of Alabama, Tuscaloosa, AL, USA, ${ }^{34}$ Universidad Autónoma del Estado de Hidalgo, Pachuca, Hgo., México, ${ }^{35}$ Department of Physics and Astronomy, University of California, Irvine, Irvine, CA, USA, ${ }^{36}$ Santa Cruz Institute for Particle Physics, University of California, Santa Cruz, Santa Cruz, CA, USA, ${ }^{37}$ Universidad de Costa Rica, San José, Costa Rica, ${ }^{38}$ Department of Physics and Mathematics, Universidad de Monterrey, San Pedro Garza García, N.L., México, ${ }^{39}$ Department of Physics, University of Maryland, College Park, MD, USA, ${ }^{40}$ Instituto de Física y Matemáticas, Universidad Michoacana de San Nicolás de Hidalgo, Morelia, Michoacán, México, ${ }^{41}$ FCFM-MCTP, Universidad Autónoma de Chiapas, Tuxtla Gutiérrez, Chiapas, México, ${ }^{42}$ Department of Physics and Astronomy, University of New Mexico, Albuquerque, NM, USA, ${ }^{43}$ University of Seoul, Seoul, Rep. of Korea, ${ }^{44}$ Universidad Politécnica de Pachuca, Pachuca, Hgo, México, ${ }^{45}$ Department of Physics, University of Wisconsin-Madison, Madison, WI, USA, ${ }^{46}$ CUCEI, CUCEA, Universidad de Guadalajara, Guadalajara, Jalisco, México, ${ }^{47}$ Universität Würzburg, Institute for Theoretical Physics and Astrophysics, Würzburg, Germany, ${ }^{48}$ Department of Physics and Astronomy, University of Utah, Salt Lake City, UT, USA, ${ }^{49}$ Department of Physics, Faculty of Science, Chulalongkorn University, Pathumwan, Bangkok 10330, Thailand, ${ }^{50}$ National Astronomical Research Institute of Thailand (Public Organization), Don Kaeo, MaeRim, Chiang Mai 50180, Thailand, ${ }^{51}$ Department of Physics, Catholic University of America, Washington, DC, USA, ${ }^{52}$ Center for Research and Exploration in Space Science and Technology, NASA/GSFC, Greenbelt, MD, USA, ${ }^{53}$ Instituto de Física Corpuscular, CSIC, Universitat de València, Paterna, Valencia, Spain 